\title{
Inventaire de la grande faune mammalienne dans la zone non protégée de Diaguiri (Kédougou, Sénégal)
}

\author{
Papa Ibnou NDIAYE*, Bathie SÈNE et Cheikh Tidiane BA \\ Département de Biologie animale, Faculté des Sciences et Techniques, \\ Université Cheikh Anta Diop, Dakar Sénégal. \\ *Corresponding author; E-mail: ibnou.ndiaye@ucad.edu.sn, Tel: 00221778142834
}

\begin{abstract}
REMERCIEMENTS
Nous tenons à remercier vivement le Ministère de l'Enseignement Supérieur et de la Recherche Scientifique du Sénégal qui nous a offert le financement qui a permis la réalisation de ce travail.
\end{abstract}

\section{RESUME}

Les effets des changements climatiques combinés aux pressions anthropiques sur les ressources naturelles sont à l'origine de la perte de la biodiversité dans les pays du Sahel comme le Sénégal, d'où l'intérêt de procéder régulièrement à une évaluation des stocks. Pour cette raison, nous avons procédé à l'inventaire des grands mammifères sauvages dans la zone non protégée de Diaguiri (Kédougou, Sénégal). Pour cela, nous avons procédé à: (i) des enquêtes préliminaires auprès des populations locales, (ii) des prospections suivant les méthodes conventionnelles des transects de reconnaissance et (iii) des piégeages photographiques. Ces méthodes ont permis de mettre en évidence la présence de 16 espèces de grands mammifères sauvages. Hormis le Chimpanzé inscrit sur la liste rouge de l'Union Internationale pour la Conservation de la Nature (UICN) dans la catégorie «En danger critique» en 2016 et le Babouin de guinée "Quasi-menacé », toutes les autres espèces répertoriées sont d'une «Préoccupation mineure ». Cependant, leurs statuts au niveau national impliquent la nécessité de renforcer les mesures de conservation pour éviter des pertes locales d'espèces. Par ailleurs, nous avons identifié les pressions anthropiques qui menacent la survie de la faune mammalienne à Diaguiri.

(C) 2018 International Formulae Group. All rights reserved.

Mots clés: Inventaire, grande faune, mammifères, Diaguiri, Sénégal.

\section{Inventory of large mammalian fauna in the unprotected zone of Diaguiri (Kedougou, Senegal)}

\begin{abstract}
Climate change effects combined with anthropogenic pressures on natural resources are causing loss of biodiversity in Sahelian countries such as Senegal, hence the need for regular stock assessment. For this reason, we conducted an inventory of large wild mammals in the unprotected area of Diaguiri (Kedougou, Senegal). For that, we proceeded with: i) preliminaries interviews in the local populations, (ii) surveys using conventional methods of reccee transects and (iii) installing camera traps. These methods have highlighted the presence of 16 large wild mammals species. Except the Chimpanzee and the Guinea Baboon which are in the
\end{abstract}


IUCN Red List and classified respectively as "Critically Endangered" in 2016 and Near-Threatened, all other species found are listed in the UICN Red List as "Least Concern". However, their statutes at national level imply the need of strengthen conservation measures to avoid local losses of these species. In addition, we have identified the anthropogenic pressures that threaten the survival of mammalian fauna in Diaguiri.

(C) 2018 International Formulae Group. All rights reserved.

Keywords: Inventory, large fauna, mammals, Diaguiri, Senegal.

\section{INTRODUCTION}

La faune sauvage africaine, et celle du Sénégal en particulier, constitue un capital mal connu qui, malheureusement, s'érode de façon alarmante. La disponibilité et la conservation des biotopes naturels est indispensable pour la survie des espèces animales. Malheureusement, ces biotopes se dégradent de jour en jour suite à la croissance démographique galopante, la déforestation et les monocultures extensives (Davies, 2017). Ces milieux naturels abritent, en effet, une communauté de grands mammifères sauvages d'une richesse exceptionnelle (Gaidet et Le Doze, 2004 ; Nobimé et al., 2008 ; Dibloni et al., 2011). Ainsi, la mise en ouvre des stratégies de conservation efficaces, dans les zones non protégées, doit se baser sur la disponibilité de données scientifiques sur la biodiversité locale et des mesures de conservation adéquates. Cependant, ces informations sont, pour la plupart, inexistantes, incomplètes ou inaccessibles aux législateurs (Sanderson et al., 2002). Or, c'est dans ces zones rurales non protégées que se joue l'avenir de la biodiversité (Gaidet et Le Doze, 2004).

Au Sénégal, peu d'études ont porté sur les grands mammifères sauvages (Niang, 2017). La plupart des travaux effectués ont porté sur les Primates, en particulier le Chimpanzé (Pruetz, 2002 ; Stewart et al., 2007; Pruetz et al., 2008; Ndiaye et al., 2013; Ndiaye et al., 2016, McGrew, 2017). Ces travaux présentent un grand intérêt pour la conservation des espèces car permettent de mieux appréhender leurs interactions entre eux et avec leurs environnements. C'est dans ce contexte que nous nous sommes engagés pour inventorier la grande faune mammalienne dans la zone non protégée de Diaguiri (Kédougou/Sénégal) pour l'identification d'aire potentielle pour la conservation.

\section{MATERIEL ET METHODES Zone d'étude}

Notre zone d'étude est circonscrite dans le premier village de Diaguiri, actuellement appelé Sinthiou Seydou (Figure 1). Elle est située à environ $50 \mathrm{~km}$ au Sud Est du Parc National du Niokolo Koba (PNNK) dans la communauté rurale de Dimboli, arrondissement de Fongolimbi, région de Kédougou. Ses coordonnées géographiques sont : 823230 Est, 1396425 Nord. Cette région est caractérisée par l'existence de deux saisons bien marquées. Une saison sèche qui dure 7 à 8 mois (d'octobre à mai) et une saison des pluies qui dure 4 mois (juin à septembre). Les moyennes annuelles de pluviométrie varient entre 1200 et $1400 \mathrm{~mm}$ de pluies. Les températures sont fortement variables avec des amplitudes comprises entre $18{ }^{\circ} \mathrm{C}$ en janvier, $38{ }^{\circ} \mathrm{C}$ en avril et dépassent très souvent les $40^{\circ} \mathrm{C}$ au mois de mai (ANSD, 2011, 2015). La végétation présente les caractéristiques des savanes boisées soudanoguinéennes immenses plus ou moins densément boisées, faisant place parfois à des forêts galeries. La nature de la savane boisée est étroitement liée aux conditions de drainage. En fonction de la position topographique, on distingue trois formations végétales :

- Une végétation ligneuse très réduite aussi bien par le nombre d'espèces que par la taille des arbres sur les pentes. Elle est essentiellement composée de Vitellaria parkii (karité), Adonsonia digitata (baobab), Bombax costatum (kapotier), Pterocarpus erinaceus (venn) et de Combretaceae.

- $\quad$ Sur le versant des cuirasses en voie de démantèlement, la végétation se diversifie 
et se densifie sur les sols graviollonnaires. Elle se caractérise par la grande diversité des espèces et par des arbres de fortes densités : Parkia biglobosa, Daniellia oliveri, Tamarindus indica, Sterculia setigera, Khaya senegalensis, entre autres.

- Dans les bas-fonds et les vallées, on trouve de grands arbres, peu nombreux, de taille supérieure à $15 \mathrm{~m}$. On y note la présence de beaucoup d'arbustes et de lianes: Combretum glutinosum, Saba senegalensis

\section{Collecte des données Enquêtes}

Nous avons effectué nos travaux de terrain entre avril 2016 et mars 2017 sur une superficie d'environ $68 \mathrm{~km}^{2}$. Nous avons procédé dans un premier temps à des enquêtes auprès des populations locales. Ces enquêtes se font sous forme d'entretiens groupés ou individuels avec la population en se basant sur un questionnaire préétabli. Nous les avons effectuées au début de l'étude et elles nous ont permis d'avoir une orientation sur les animaux susceptibles d'être rencontrés dans la zone, les endroits les plus fréquentés par ces animaux, l'évolution des effectifs dans le temps et les relations entre les populations humaines et la faune sauvage. Les personnes enquêtées sont pour la plus part des chasseurs. Ce qui nous a permis d'avoir des informations très utiles pour l'orientation de nos prospections.

\section{Prospections}

Au cours des prospections, nous avons utilisé la méthode des transects de reconnaissance (Kühl et al., 2008) pour procéder à des observations directes et/ou indirectes des indices de présence des grands mammifères sauvages. C'est aussi pendant ces prospections que nous identifions les endroits stratégiques pour y installer les pièges photographiques. La méthode des transects de reconnaissance consiste à parcourir des bandes de largeurs variables (10 à 20 mètres pour cette étude) en fonction de la visibilité des observateurs. La longueur des bandes est généralement comprise entre 5 et 8 Kilomètres en moyenne pour notre étude. La détermination des bandes s'est faite à l'aide des cartes de la zone et des informations disponibles pendant la période préparatoire des missions. Nous avons choisi les transects de sorte que toute la zone d'étude soit couverte (Figure 2). Les prospections sont de types pédestre et débutent le matin de bonne heure (entre 06 h-07 h) jusqu'en début d'après-midi (souvent entre $15 \mathrm{~h}-16 \mathrm{~h}$ ). Parfois, les transects sont parcourues l'aprèsmidi entre 16 heures et 20 heures.

\section{Pièges photographiques}

Cette méthode permet, même dans des conditions extrêmes (vent, pluie,...), de prendre des images aussi bien le jour que la nuit sans perturber les activités des animaux. L'appareil est le plus souvent fixé sur un tronc d'arbre dans des endroits stratégiques, comme les points d'eau, les couloirs de passage ou à l'intérieur des galeries forestières qui constituent les biotopes de prédilections de nombreux animaux de la zone d'étude. Ainsi, un total de 20 pièges photographiques à infrarouge a été déployé à travers la zone d'étude pendant 135 jours soient 3240 heures de fonctionnement. Les appareils sont munis de capteurs sensibles aux mouvements d'animaux qui traversent leurs objectifs dans un champ de 20 à 25 mètres (plage de détection). Ils sont paramétrés afin de prendre une photo en couleur toutes les 10 secondes (intervalle/retard) et sur chacune d'elles apparaît : la date, l'heure, et la température du milieu ambiant au moment de la prise. Les appareils fonctionnaient 24 heures sur 24 et des LED (light-emitting diode) infrarouge fournissaient l'éclairage pour une bonne et nette prise d'images pendant la nuit ou en condition d'obscurité. Sachant qu'il existe une relation entre richesse spécifique et superficie étudiée (Marinosci, 2010), nous avons couvert le maximum de surface possible pour avoir de meilleurs résultats.

\section{RESULTATS \\ Enquêtes}

Il est apparu au cours de nos entretiens avec les populations locales que les espèces les plus fréquentes dans cette zone appartiennent aux ordres des Primates et des Artiodactyles, principalement les Babouins de guinée (en peulh : Gookirou), les singes verts 
(en peulh: Wandou Bodé), les phacochères (en peulh: Mbaba Ladé), les Guibs harnachés (en peulh: Diguré ou Mbaldé). Les espèces comme le Chimpanzé (Démorou), les Hyènes (en peulh: Bonorou) et les Antilope cheval (Koba) y existent mais sont très difficiles à voir. Les lions (en peulh: Ngayérou) ont disparu de la zone.

\section{Répertoire des espèces de grands mammifères sauvages observées à Diaguiri}

Nous présentons dans cette partie une liste qualitative des espèces de grands mammifères sauvages que nous avons rencontrés directement et/ou indirectement durant notre inventaire (Tableau 1, Figures 3, 4 et 5).

Nous avons répertorié au total, 16 espèces réparties dans 16 genres, 12 familles et 4 ordres (Tableau 1). Parmi ces 16 espèces, nous avons pu obtenir des images de 10 espèces à l'aide des pièges photographiques déployés dans la zone d'étude. Ces images concernent le Chimpanzé (Figure 3 : Photos 1 à 3 ), le Babouin de guinée (Figure 3 : Photos 4 et 5), le Singe vert (Figure $3:$ Photos 6 et 7), le Guib harnaché (Figure 4 : Photos 8 et 9), le Phacochère (Figure 4: Photo 10), la Mangouste des marais (Figure 4: Photo 11), la Genette commune (Figure 4 : Photo 12), la Civette d'Afrique, le Chacal (Figure 5: Photos 14 et 15) et le Porc épic (Figure 5 : 16). Pour les 6 autres espèces, notamment le Chat sauvage (Felis sylvestris), la Mangouste à queue blanche (Ichneumia albicauda), la Hyène (Crocuta crocuta), l'Antilope cheval (Hippotragus equinus) et le Grand aulacode (Thryonomys swinderianus) et le Patas (Erythrocebus patas), nous avons procédé à des observations directes et/ou de leurs indices (Tableau 2).

L'ordre des Carnivores apparaît comme étant le plus représenté avec 7 espèces (Canis sp, Crocuta crocuta, Felis sylvestris, Ichneumia albicauda, Atilax paludinosus, Genetta genetta, Civettictis civetta), ensuite les Primates avec 4 espèces (Pan troglodytes verus, Cercopithecus a. sabaeus, Papio papio, Erythrocebus patas), suivi par les Artiodactyles avec 3 espèces (Hippotragus equinus, Phacochoerus africanus, Tragelaphus scriptus) et enfin les Rongeurs avec 2 espèces (Hystrix cristata, Thryonomys swinderianus). Nous avons décompté au cours de l'étude 11 individus de chimpanzés ( $P . t$. verus) dont 2 juvéniles, 25 individus de babouins de guinée ( $P$. papio) et 23 individus de patas $(E$. patas). Pour les autres espèces, les effectifs variaient entre 2 et 5 individus.

\section{Menaces anthropiques sur la survie des espèces}

Les menaces qui pèsent sur la biodiversité sauvage de Diaguiri sont d'ordre divers (Figures 6 et 7). Elles peuvent être directes à travers la chasse ou indirectes comme les feux de brousse, la déforestation, le pâturage par les Hagas (Peulh), l'exploitation de la sève de Borassus aethiopum par les Bassaris pour obtenir du vin (Bandji) et l'orpaillage.

- La chasse pratiquée par les populations locales dans la zone est de type traditionnel c'est-à-dire liée à la subsistance.

- Les feux de brousses sont utilisés par les populations pour un accès facile et une meilleure visibilité sur le terrain pour leurs déplacements. Ces feux sont catégorisés en deux types : les feux précoces et les feux tardifs. Ces derniers sont considérés comme l'une des principales causes de la dégradation des ressources naturelles de la zone car entrainent la mort des essences ligneuses sensibles et la disparition de la biomasse herbacée qui constitue l'aliment de base pour de nombreux Artiodactyles de la zone (guib et antilope cheval).

- La déforestation est matérialisée dans la zone par l'abatage d'arbres comme Pterocarpus erinaceus, Diospyros mespiliformis, Anogeissus leicarpus et Khaya senegalensis qui jouent un rôle important pour la survie de certains groupes de primates sympatriques comme le chimpanzé. Cette déforestation est surtout liée à la recherche de terre de cultures, de bois d'œuvre (constructions, mobiliers), à la récolte de miel et à l'implantation de site d'orpaillage.

- Le Khaya senegalensis inscrit dans la liste rouge de l'UICN en catégorie d'espèce « 
Vulnérable »(VU) est très prisé par les transhumants (Hagas) pour le pâturage.

- L'exploitation abusive de la sève des rôniers (Borassus aethiopium) par certaines populations qui s'en servent comme boisson alcoolisée (Bandji) entraine une disparition progressive de cette espèce dans la zone.

Tableau 1: Liste qualitative des espèces répertoriées à Diaguiri.

\begin{tabular}{|c|c|c|c|c|}
\hline & Famille & Espèce & Nom français & Nom Peulh \\
\hline \multirow[t]{3}{*}{ Artiodactyles } & Bovidae & Tragelaphus scriptus & Guib harnaché & Diguré/Mbaldé \\
\hline & Hyppotraginae & Hippotragus equinus & Antilope cheval & Koba \\
\hline & Suidae & Phacochoerus africanus & Phacochère commun & Mbaba ladé \\
\hline \multirow[t]{7}{*}{ Carnivores } & Canidae & Canis sp & Chacal & Donndu \\
\hline & Viveridae & Genetta genetta & Genette commune & Balérou \\
\hline & & Civettictis civetta & Civette d'Afrique & Mbouta kerké \\
\hline & Hyenidae & Crocuta crocuta & Hyène tachetée & Bonorou \\
\hline & Felidae & Felis silvestris & Chat sauvage & Kotourou \\
\hline & & Atilax paludinosus & Mangouste des marais & Bambarou \\
\hline & Herpestidae & Ichneumia albicauda & Mangouste à queue blanche & Bambarou \\
\hline \multirow[t]{4}{*}{ Primates } & & Cercopithecus a. sabaeus & Singe vert & Wandou balé \\
\hline & Cercopithecinae & Erythrocebus patas & Singe rouge ou Patas & Wandou bodé \\
\hline & & Papio papio & Babouin de Guinée & Gookirou \\
\hline & Pongidae & Pan troglodytes verus & Chimpanzé & Démorou \\
\hline \multirow[t]{2}{*}{ Rongeurs } & Hystricidae & Hystrix Cristata & Porc-épic & Sangaldé \\
\hline & Thryonomyidae & Thryonomys swinderianus & Grand aulacode & Magnaré \\
\hline
\end{tabular}

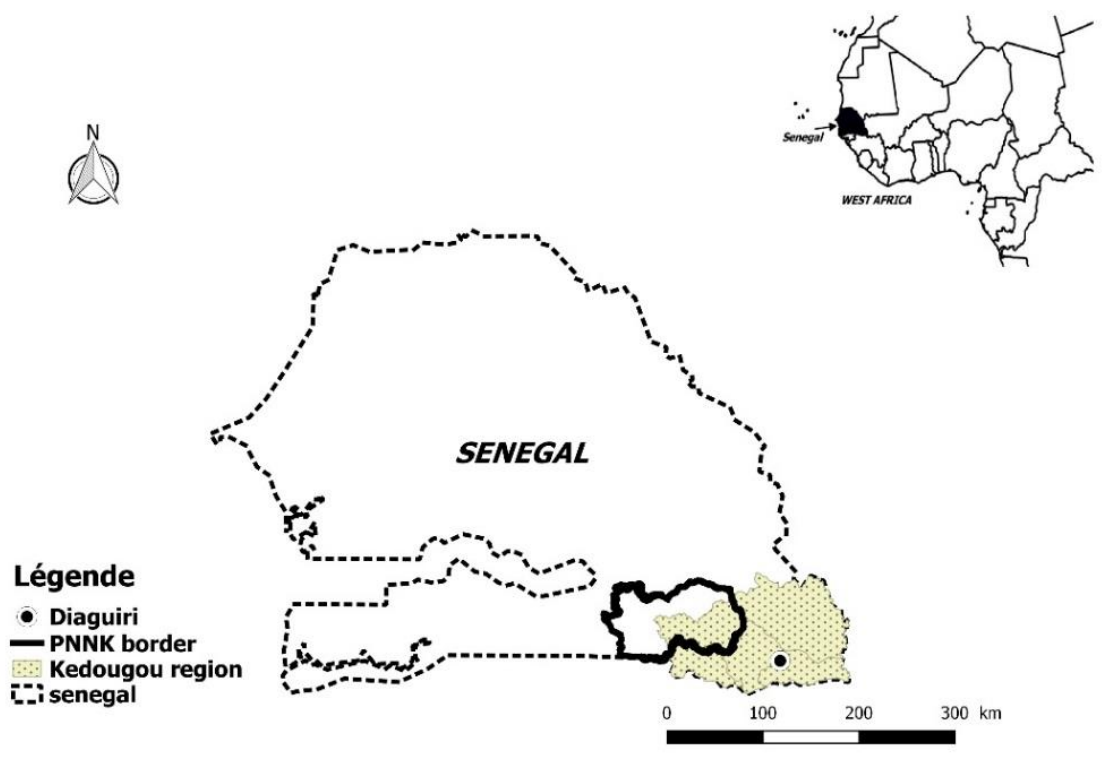

Figure 1: Localisation de la zone d'étude. 


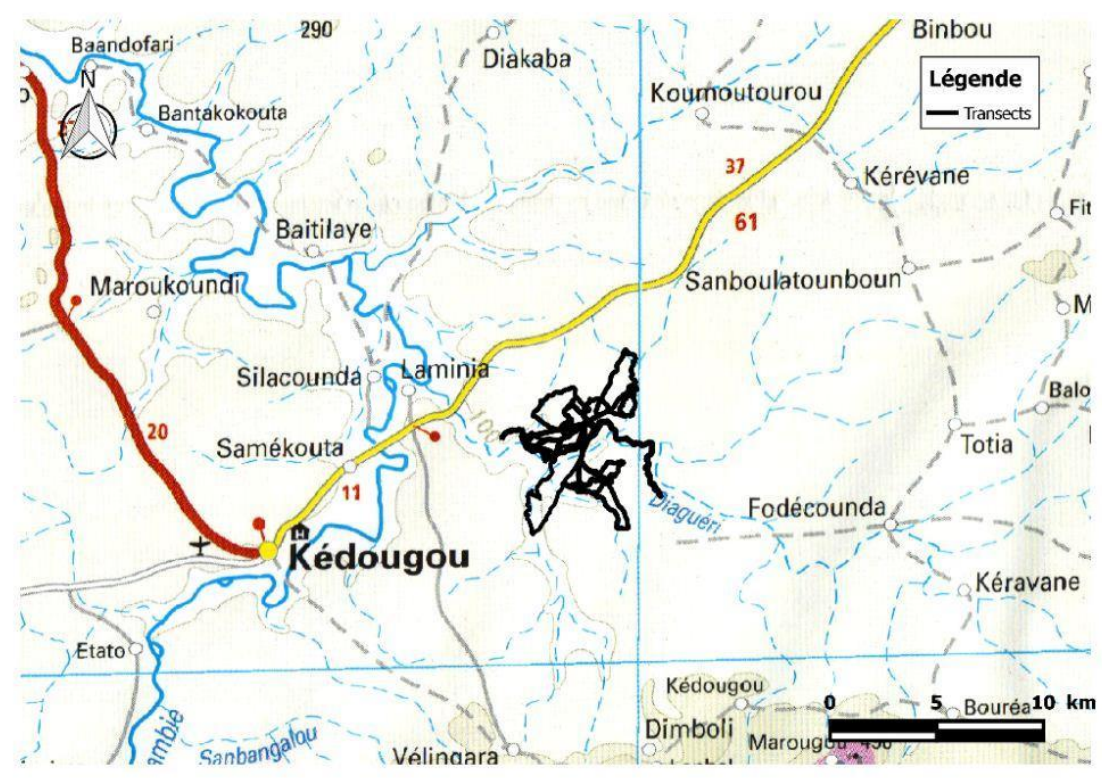

Figure 2: Localisation des transects de reconnaissance effectués.
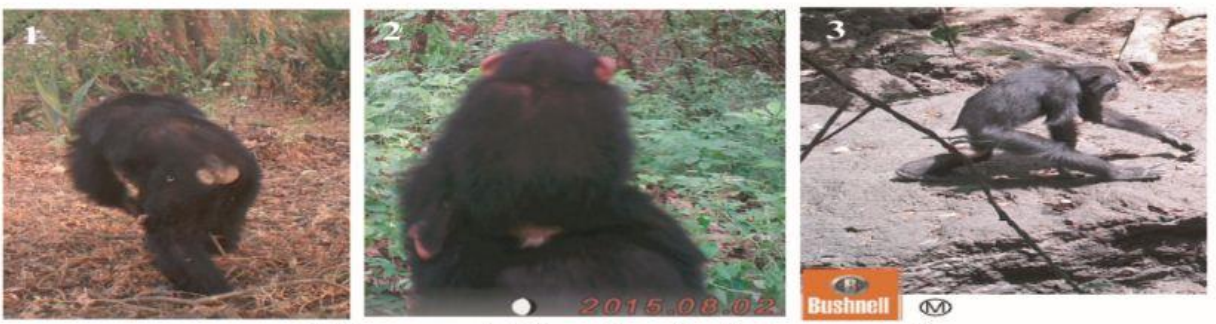

Chimpanzé (Pan troglodytes verus)
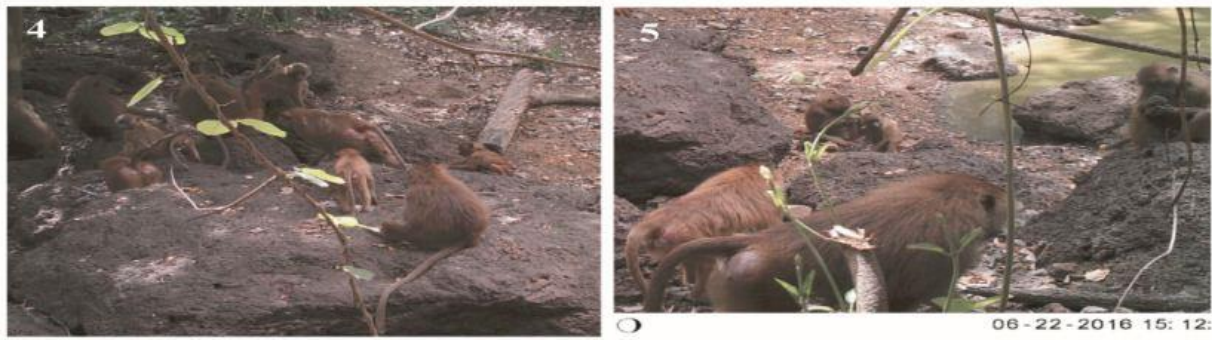

Babouin de guinée (Papio papio)
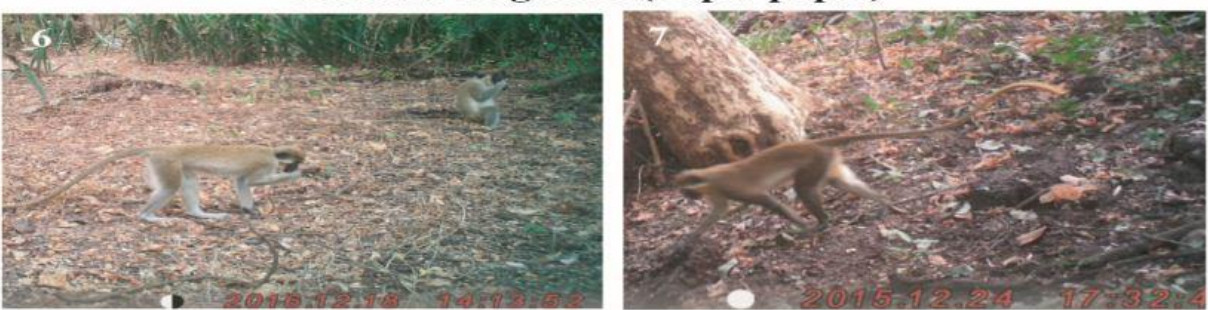

Singe vert (Cercopithecus aethiops)

Figure 3 : Images d'espèces de mammifères obtenues à l'aide des pièges photographiques. Photos 1,2 et 3 : Chimpanzé ; Photos 4 et $5:$ Babouin de guinée ; Photos 6 et $7:$ Singe vert. 

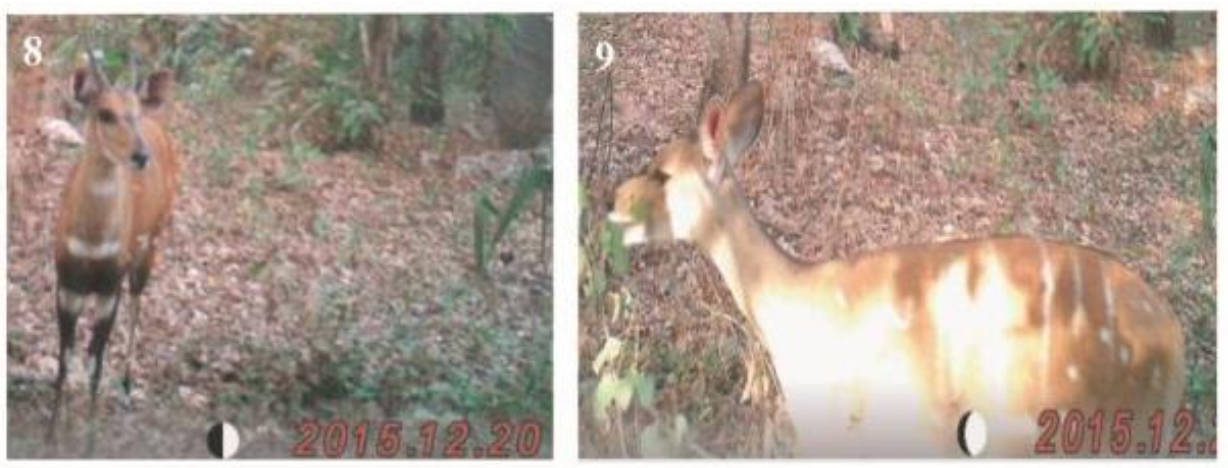

\section{Guib harnaché (Tragelaphus scriptus)}

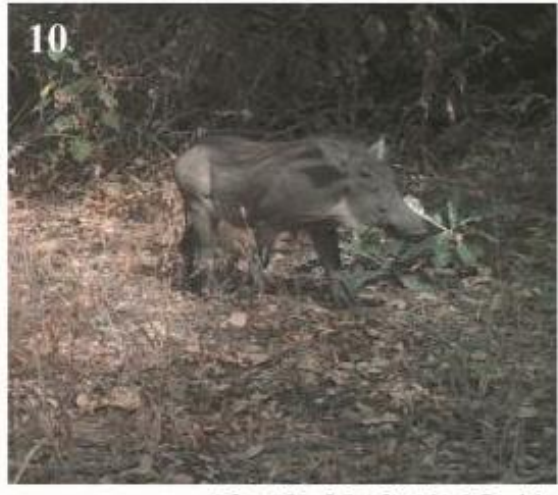

\section{Phacochère}

12 - 16 - 2016 10: 05: 40

\section{(Phacocherus africanus)}

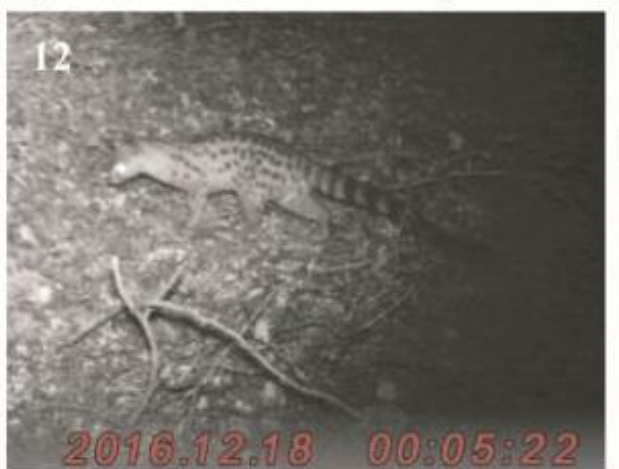

Genette commune

(Genetta genetta)

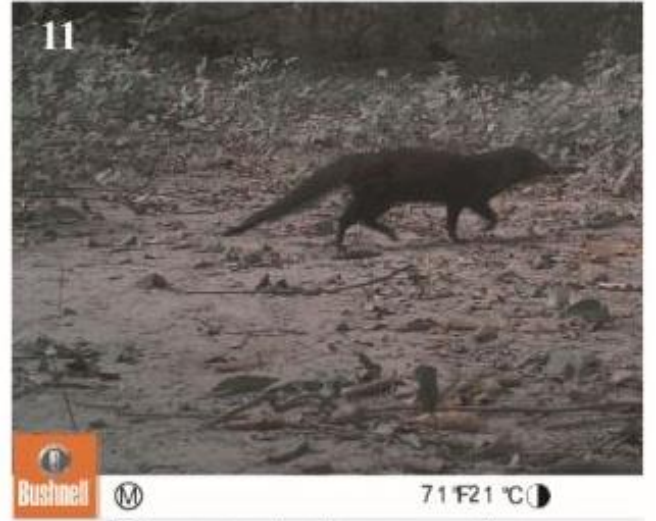

Mangouste des marais (Atilax paludinosis)

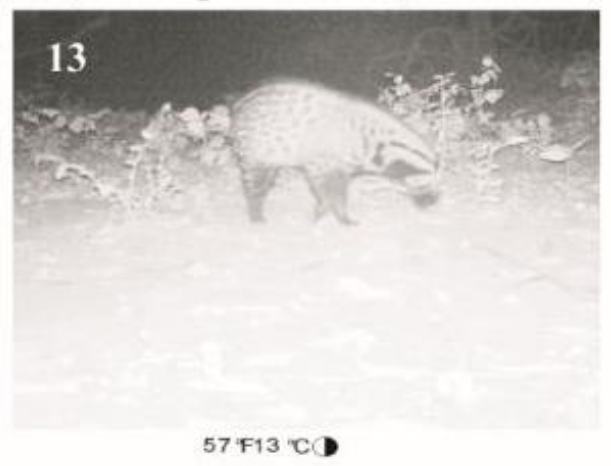

Civette d'afrique

(Civettictis civetta)

Figure 4: Images d'espèces de mammifères obtenues à l'aide des pièges photographiques. Photo 8 : Guib harnaché mâle ; Photo 9: Guib harnaché femelle ; Photo 10 : Phacochère ; Photo 11 : Mangouste des marais ; Photo 12 : Genette ; Photo 13: Civette d'Afrique. 

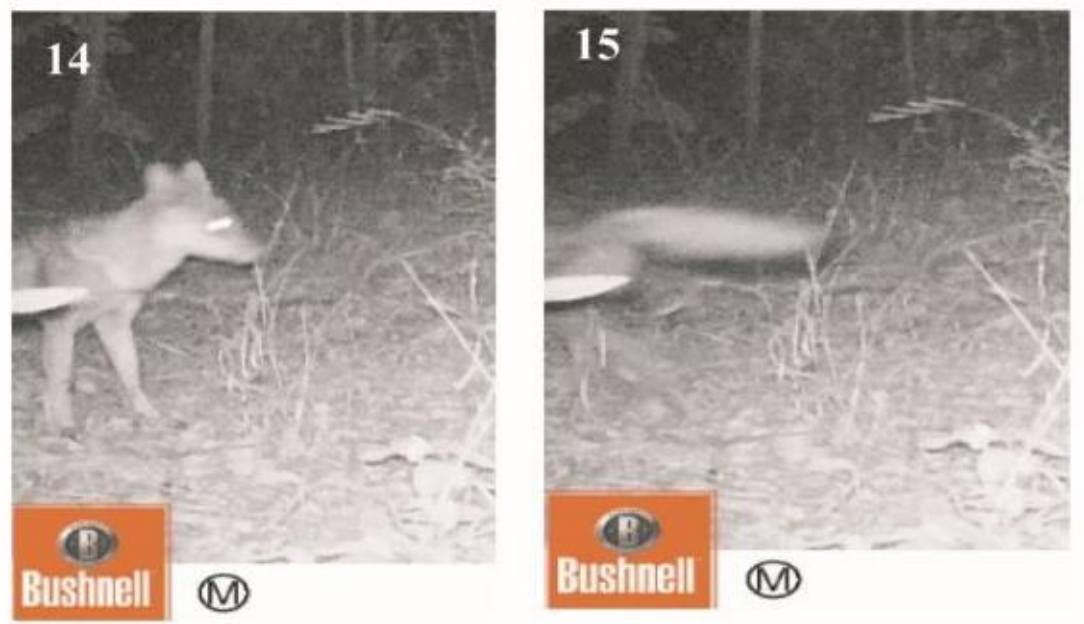

\section{Chacal à flancs rayés (Canus adustus)}

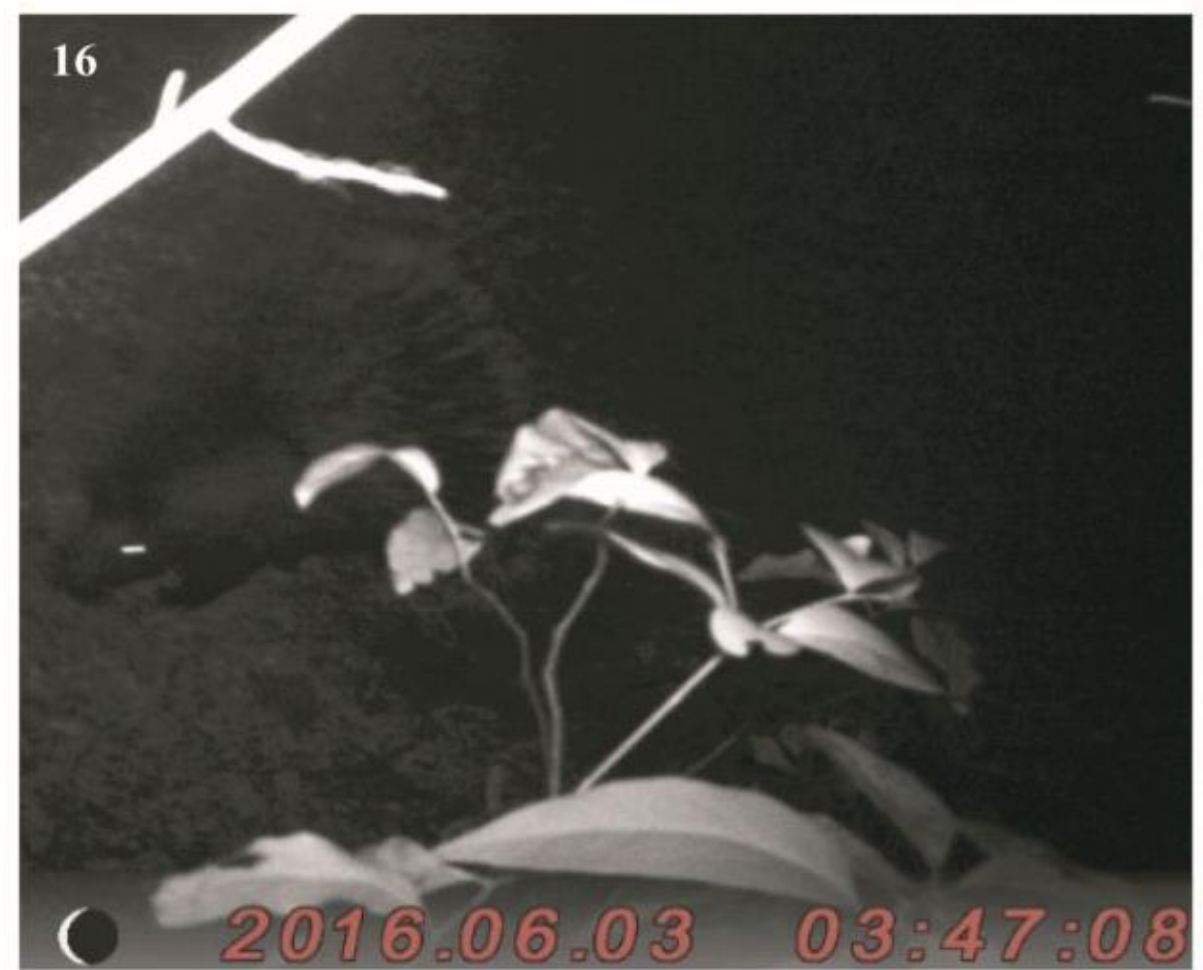

\section{Porc épic (Hystrix cristata)}

Figure 5: Images d'espèces de mammifères obtenues à l'aide des pièges photographiques. Photo 14 : la tête et une partie du tronc du Chacal à flanc rayé ; Photo 15 : la queue du Chacal à flanc rayé ; Photo 16 : Porc épic. 


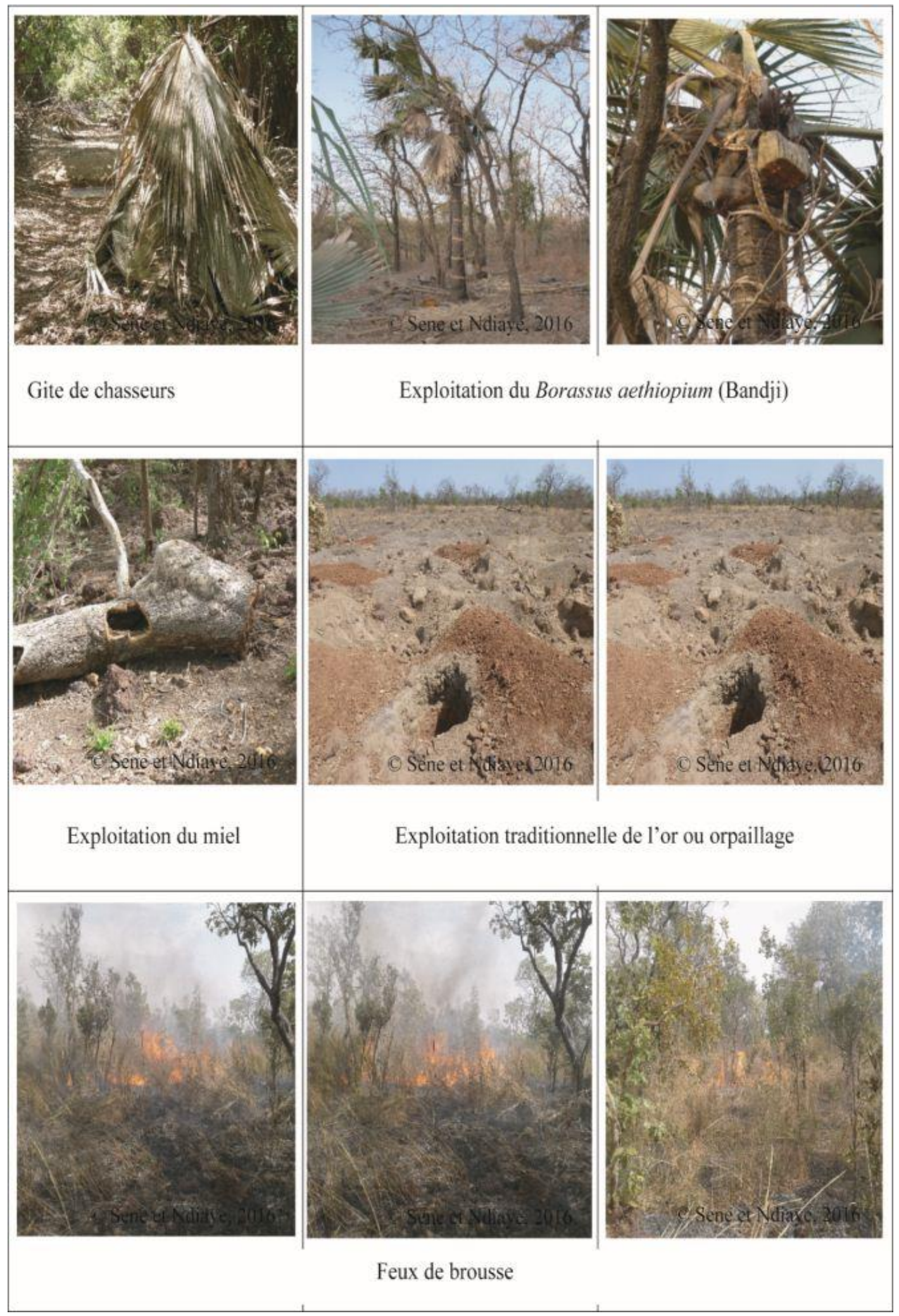

Figure 6: Répertoire des menaces anthropiques sur la survie de la faune à Diaguiri. 


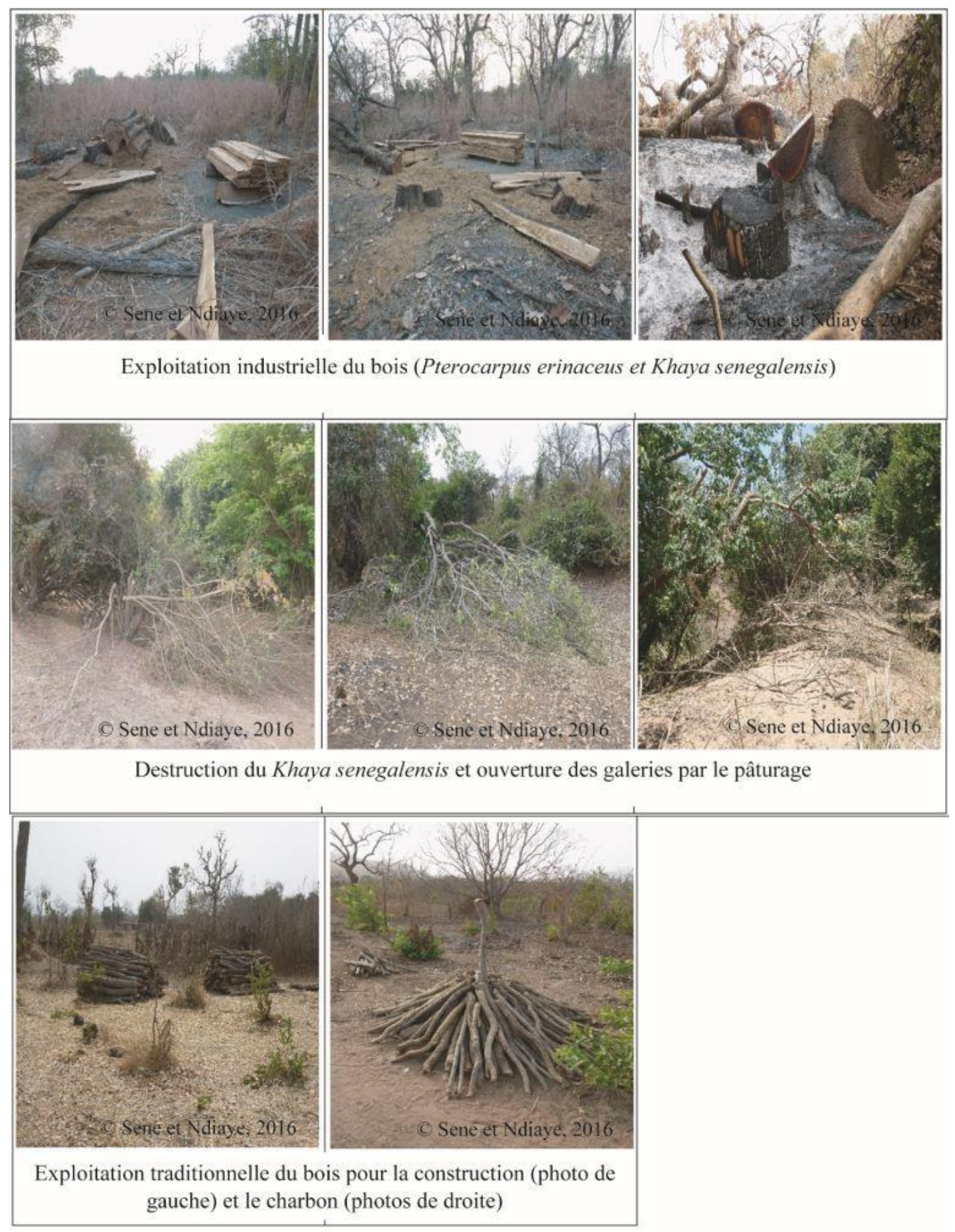

Figure 7: Répertoire des menaces anthropiques sur la survie de la faune à Diaguiri. 
Tableau 2: Localisation d'observations d'espèces non capturées par les pièges photographiques.

\begin{tabular}{lccccc}
\hline Dates & Heures & Espèces & Types d'observation & Latitude & Longitude \\
\hline $04 / 04 / 2016$ & $10 \mathrm{~h} 04$ & Ichneumia albicauda & directe & 1392462 & 824173 \\
\hline $07 / 04 / 2016$ & $06 \mathrm{~h} 59$ & Crocuta crocuta & Empreintes et crottes & 1766434 & 0469078 \\
\hline $11 / 04 / 2016$ & $07 \mathrm{~h} 07$ & Felis sylvestris & directe & 1395260 & 822934 \\
\hline $02 / 06 / 2016$ & $09 \mathrm{~h} 56$ & Thryonomys swinderianus & directe & 1396554 & 824373 \\
\hline $07 / 06 / 2016$ & $08 \mathrm{~h} 42$ & Hippotragus equinus & directe & 1398521 & 824676 \\
\hline $17 / 12 / 2016$ & $12 \mathrm{~h} 42$ & Erythrocebus patas & directe & 1392652 & 824752 \\
\hline
\end{tabular}

\section{DISCUSSION}

Les résultats de cette étude nous donnent, pour une première fois, une information globale sur la biodiversité mammalienne dans la Zone d'Intérêt Cynégétique de Diaguiri. Ainsi, l'évaluation qualitative des grands mammifères sauvages de Diaguiri a révélé une importante biodiversité de grands mammifères sauvages avec la présence de 16 espèces composées en majorité de Carnivores et de Primates. Nous avons identifié parmi ces espèces le chimpanzé (Pan troglodytes verus) considéré comme l'espèce phare de la région eu égard à son statut : «En danger critique » et le babouin de guinée (Papio papio) qui a un statut «Quasi-menacé »(UICN, 2016). Les autres espèces observées au cours de notre étude $(C . s p, C$. crocuta, $F$. silvestris, $C$. civetta, G. genetta, I. albicauda, A. paludinosus, T. swinderianus, $H$. cristata, $H$. equinus, P. africanus, E. patas, C. a. sabaeus, T. scriptus) sont considérées comme étant de «Préoccupation mineure » par l'UICN mais leurs statuts au niveau national impliquent la nécessité de renforcer les mesures de conservation pour éviter des pertes d'espèces au niveau local. L'utilisation des pièges photographiques a été déterminante dans ce travail car elle a permis de mettre en évidence par des images photographiques la présence d'espèces très difficiles à voir dans cette région. C'est le cas du chimpanzé et de certaines espèces à mœurs nocturnes comme le Porc epic, la Genette, le Chacal et la Civette d'Afrique. Ces résultats confirment donc les affirmations de Kierulff et al. (2004), Tan et al. (2013) et Pebsworth et Lafleur (2014) qui pensent que la méthode des pièges photographique est d'une grande utilité pour l'étude des mammifères, en particulier les espèces rares et permet de faire le suivi des espèces sans avoir besoin de les observer directement ou d'utiliser des pièges pour la capture physique comme cela se faisait avant.

Diaguiri n'est pas une zone protégée mais est dotée de potentialités énormes au point de vue de la biodiversité. Ceci est surtout favorisé par sa position dans la zone de transition entre le Parc National du Niokolo Koba au Sénégal et le Parc National de Badiar en Guinée et la disponibilité de biotopes naturels et d'eau. Cependant, si des mesures adéquates de gestion des ressources naturelles (faune, flore, mines, etc.) ne sont pas prises, cela pourra engendrer des dommages naturelles allant jusqu'à une perte de biodiversité. Les menaces répertoriées au cours de cette étude montrent une forte pression anthropique sur les ressources naturelles de Diaguiri. Ce qui peut compromettre le devenir de la diversité biologique dans cette zone. D'où la nécessité de mettre en place des plans adéquats de gestion des ressources comme c'est le cas ailleurs pour une meilleurs conservation de la biodiversité (Bitty et al., 2013; Tsakem et al., 2015). Selon Koumba et al. (2016), le trafic illégal de la faune sauvage est actuellement la plus grande menace pour l'intégrité de l'environnement. La consommation de la viande sauvage en Afrique est aussi aujourd'hui l'une des menaces les plus accentuées pour les grands vertébrés (Fa et al., 
2006 ; Linder et Oates, 2011). Ainsi, le renforcement des législations nationales de la Convention sur le Commerce International des Espèces de faune et de flore sauvages menacées d'extinction (CITES) peut aider à la conservation des ressources. Les habitats de la faune sauvage sont perdus de plus en plus en Afrique parce qu'ils sont convertis en terres agricoles, en lieux de résidence, en réseaux de transports et en industries extractives (Wilkie et al., 2016). Pour ces auteurs, le commerce de viande sauvage est une activité économique très attractive pour les braconniers.

\section{Conclusion}

Au vue de l'importance de la diversité de la faune mammalienne à Diaguiri, en particulier les espèces de primates et la forte pression anthropique sur les ressources naturelles, nous pensons que les décideurs doivent mettre en place des campagnes de sensibilisation auprès de ces populations, favoriser la mise en place de nouvelles formes d'exploitations économiques en adéquation avec une gestion rationnelle des ressources naturelles et mettre en place des plans de gestion communautaires des ressources naturelles.

\section{CONFLIT D'INTERETS}

Les auteurs déclarent qu'il n'existe aucun conflit d'intérêts pour cet article.

\section{CONTRIBUTIONS DES AUTEURS}

PIN et BS ont fait le travail de terrain et ont rédigé le manuscrit. CTB a contribué à la rédaction du manuscrit.

\section{REMERCIEMENTS}

Nous associons à ces remerciements les autorités de l'Université Cheikh Anta Diop, en particulier le Doyen de la Faculté des Sciences et Techniques pour avoir autorisé à chaque fois notre absence pour effectuer les travaux de terrain et mis à notre disposition un véhicule $4 \mathrm{X} 4$. Nos remerciements vont aussi à l'endroit des populations locales de Diaguiri pour leur collaboration.

\section{REFERENCES}

ANSD. 2011. Agence national de la statistique et de la démographie. Situation Economique et Sociale Régionale de Kédougou 2010, 95p.

ANSD. 2015. Agence national de la statistique et de la démographie. Situation Economique et Sociale Régionale de Kédougou 2013, 91p.

Bitty EA, Kadjo B, Gonedele Bi S, Okon MO, Kouassi PK. 2013. Inventaire de la faune mammalogique d'une forêt urbaine, le Parc National du Banco, Côte d'Ivoire. Int. J. Biol. Chem. Sci., 7(4): 1678-1687. DOI : http://dx.doi.org/10.4314/ijbcs.v7i4.23.

Marinosci C. 2010. Atlas régional des mammifères sauvages : méthodologie pour une étude de faisabilité dans la région Provence-Alpes-Côte d'Azur. Mémoire de Master, Université Paul Cézanne, 66p.

Davies J. 2017. Biodiversité et Grande Muraille Verte: Gérer la Nature pour un Développement Durable au Sahel. UICN : Ouagadougou, Burkina Faso ; 66.

Dibloni OT, Millogo AN, Ouedraogo AJ, Guenda W, Vermeulen C. 2011. Diversité faunique et distribution des activités de braconnage dans la réserve de biosphère de la mare aux hippopotames au Burkina Faso. Int. J. Biol. Chem. Sci., 5(5): 18131827.

DOI: http://dx.doi.org/10.4314/ijbcs.v5i5.5

Fa JE, Seymour S, Dupain J, Albrechtsen L, Macdonald D. 2006. Getting to grips with the magnitude of exploitation: bushmeat in the Cross-Sanaga rivers región, Nigeria and Cameroon. Biol. Conserv., 129: 497510.Doi.org/10.1016/j.biocon.2005.11.031

Gaidet N, Le Doze S. 2004. Indicateurs de pression environnementale selon un degré d'anthropisation croissante; La Gestion Pratique de la Faune en Zone Communale au Zimbabwe. CIRAD, 54p.

UICN 2016. The IUCN Red List of Threatened Species. IUCN: Gland, Switzerland.

Koumba Pambo AF, Carroll T, Lelanchon L, Ehi-Ebewele E, Sonko A, White L. 2016. International trade in endangered species: the challenges and successes of the $17^{\text {th }}$ conference of parties to the convention on 
international trade in endangered species of wild fauna and flora (CITES). Afr. $J$. Ecol., 54: 399-401. Doi: 10.1111/aje.12394

Kierulff MC, Santos GR, Canale GR, Guidorizzi CE, Cassano CR. 2004. The use of camera-traps in a survey of the buffheaded capuchin monkey, Cebus xanthosternos. Neotrop. Primates, 12: 5659.

Linder JM, Oates JF. 2011. Differential impact of bushmeat hunting on monkey species and implications for primate conservation in Korup National Park, Cameroon. Biol. Conserv., 144: 738-745. Doi: 10.1016/j.biocon.2010.10.023

McGrew WC. 2017. Field studies of Pan troglodytes reviewed and comprehensively mapped, focusing on Japan's contribution to cultural primatology. Primates, 58(1): 237-258. Doi.org/10.1007/s10329-0160554-y

Ndiaye PI, Badji L, Lindshield SM, Bâ CT, Pruetz J. 2016. Etude préliminaire sur la distribution du chimpanzé de l'Afrique de l'ouest, Pan troglodytes verus (Schwarz, 1934), dans la zone non protégée de Diaguiri (Kédougou, Sénégal): Implication pour sa conservation. Communication au 29eme colloque de la Société Française de Primatologie, Marseilles (France).

Ndiaye PI, Gérard G, Galat-Luong A, Nizinski G. 2013. Note on the seasonal use of lowland and highland habitats by the West African Chimpanzee Pan troglodytes verus (Schwarz, 1934) (Primates: Hominidae): Implications for its conservation. J. Threat. Taxa, 5(2): $3697-$ 3700. http://dx.doi.org/10.11609/JoTT. 03229.3229.3697-700.

Niang A. 2017. Inventaire des grands mammifères sauvages dans la zone de reboisement de la grande muraille verte au Ferlo (Nord, Sénégal). Mémoire de master 2 de Biologie Animale, Dakar, Université Cheikh Anta Diop de Dakar, Faculté des Sciences et techniques, $33 \mathrm{pp}$.
Nobimé G., Gaoué OG, Sinsin B. 2008. Distribution des espèces de primates au Bénin et ethnozoologie. Int. J. Biol. Chem. Sci., 2(3): 346-354.

Pebsworth P, Lafleur M. 2014. Advancing primate research and conservation through the use of camera traps: introduction to the special issue. Int. J. Primatol., 34(5): 825840. DOI: $10.1007 / \mathrm{s} 10764-014-9802-4$.

Pruetz JD. 2002. Competition between chimpanzees (Pan troglodytes verus) and humans in southeastern Senegal. Am. J. Physiol. Anthr., 34: 128. DOI: 10.1002/ajpa.20014.

Pruetz JD, Fulton SJ, Marchant LF, McGrew WC, Schiel M, Waller M. 2008. Arboreal nesting as anti-predator adaptation by Savanna Chimpanzees (Pan troglodytes verus) in southeastern Senegal. Am. J. Primatol., 70(4): 393-401. Doi 10. 1002/ajp.20508.

Sanderson J, Barrie A, Coleman J E, Kante S, Ouattara S, Toukara EHO. 2002. Inventaire rapide des grands mammifères des Forêts Classées de la Haute Dodo et du Cavally en Côte d'Ivoire, $117 \mathrm{p}$.

Stewart FA, Pruetz JD, Hansell MH. 2007. Do Chimpanzees build comfortable nests? Am. J. Primatol., 69: 930-939. DOI 10.1002/ajp.20432.

Wilkie DS, Wieland M, Boulet H, Le Bel S, Vliet Nv, Cornelis D, Warnon VB, Nasi R, Fa JE. 2016. Eating and conserving bushmeat in Africa. Afr. J. Ecol., 54: 402414. DOI: 10.1111/aje.12392.

Tan CL, Yang Y, Niu K. 2013. Into the night: Camera traps reveal nocturnal activity in a presumptive diurnal primate, Rhinopithecus brelichi. Primates, 54 : 16.DOI : 10.1007/s10764-014-9802-4.

Tsakem SC, Tchamba M, Weladji RB. 2015. Les gorilles du Parc National de Lobéké (Caméroun): interactions avec les populations locales et implications pour la conservation. Int. J. Biol. Chem. Sci., 9(1): 270-280.

DOI: http://dx.doi.org/10.4314/ijbcs.v9i1.24. 